Res Publica. Revista de Historia de las Ideas Políticas ISSN: $1576-4184$

http://dx.doi.org/10.5209/RPUB.56616

\title{
¿Teorías "energéticas" de la cultura? La inmisericorde crítica de Weber al ganador del Premio Nobel de Química (1909)*
}

\author{
Eduardo Zazo Jiménez ${ }^{1}$
}

Teorías "energéticas" de la cultura es una reseña escrita en 1909 por Weber. El libro reseñado se titula Energetische Grundlagen der Kulturwissenschaft ${ }^{2}$ [Fundamentos energéticos de la ciencia de la cultura] y fue escrito por el Premio Nobel de Química Wilhelm Ostwald y publicado también en 1909. La reseña de Weber es demoledora, prolija en adjetivos descalificativos y no exenta de toques irónicos y mordaces. En este inusual escrito Weber se despacha contra el diletantismo de un científico natural que considera que su método "energético", exitoso en el ámbito de la química, puede aplicarse sin más al ámbito de las ciencias culturales. Entonces un Weber metodológico realiza una crítica destructiva conminando a Ostwald a dedicarse a sus asuntos, como si dijera "callen los científicos naturales en asuntos ajenos".

Ningún historiador, ningún economista-nacional, ningún representante de las disciplinas científico-culturales tiene hoy la arrogancia de prescribir a los químicos y a los tecnólogos qué métodos y qué planteamientos tendrían que emplear. Que los representantes de estas últimas disciplinas aprendan a ser igualmente modestos — esto es el presupuesto de una colaboración fructífera, que nadie desea más que quien esto escribe ${ }^{3}$.

Como es habitual en Weber, su propuesta posee una carga normativa enorme que es preciso desgranar. Además, se trata del único diálogo "metodológico" de Weber con un científico ajeno a las ciencias de la cultura — en este caso, con un químico. A pesar de constituir una reseña, el texto es rico en reflexiones sobre la especificidad de las ciencias de la cultura, sobre la distinción entre hechos y juicios de valor y sobre las ingenuas defensas, por parte de científicos tanto "naturales" como "culturales" o "sociales", de la unidad del método científico. En este breve texto de presentación intentaremos, de forma concisa: 1) ubicar temáticamente este texto en el caótico con-

\footnotetext{
Al acabar este texto de presentación el lector encontrará la versión castellana del texto de Weber Teorías "energéticas” de la cultura («Energetische» Kulturtheorien). Las citas del texto traducido irán, a partir de ahora, indicadas con "infra”. Hasta donde sé, se publica por primera vez en castellano. La traducción ha sido realizada a partir del texto M. Weber, “«Energetische» Kulturtheorien”, M. Weber, Gesammelte Schriften zur Wissenschaftslehre 6 , Tübingen, J. C. B. Mohr (Paul Siebeck), 1985, pp. 400-426. Agradezco a Roberto Navarrete Alonso la revisión de la traducción y sus valiosas sugerencias.

1 eduardo.zazo.jimenez@gmail.com Universidad Autónoma de Madrid

2 W. Ostwald, Energetische Grundlagen der Kulturwissenschaft, Leipzig, W. Klindhardt, 1909.

3 Infra, p. 467.
} 
junto de la obra weberiana como escrito metodológico; 2) analizar la recepción "metodológica" de Weber y la de los escritos "metodológicos" en general; 3) describir la novedad, el interés y las ideas centrales del texto, mostrando su especial relevancia para la comprensión del postulado de la Wertfreiheit.

\section{Ubicación de Teorías "energéticas" de la cultura}

De acuerdo con la asentada tradición de estudios sobre Weber, este texto pertenecería a los escritos metodológicos ${ }^{4}$. En cuanto tal, podría ser descrito como el último de los textos redactados por Weber a lo largo de ese período de seis años (1903-1909) de intensa dedicación a las cuestiones metodológicas ${ }^{5}$. Durante este período Weber escribe sus textos sobre Roscher y Knies, el artículo sobre la "objetividad", sus críticas de Meyer y Stammler y las reseñas de Brentano y de Ostwald ${ }^{6}$. Salvo este último, el resto de textos se encuentran ya accesibles en castellano ${ }^{7}$. Es lícito y oportuno preguntarse por qué este texto no había sido traducido a nuestra lengua. La razón es evidente: se trata de un texto menor (una reseña), con una escritura caótica, pocos desarrollos teóricos y sobre un autor (Ostwald) y unas cuestiones (el energetismo) completamente olvidados desde hace mucho tiempo. No obstante, este texto muestra, como he indicado y como veremos más adelante, algunos rasgos interesantes sobre asuntos centrales del pensamiento de Weber.

Teorías "energéticas" de la cultura pertenece a los escritos metodológicos, especialmente a la fase comprendida entre 1903 y 1909. Ahora bien, durante esos años Weber no se dedica exclusivamente a la redacción de escritos sobre metodología.

Tradición que se remonta a la publicación en 1922 de los Gesammelte Aufsätze zur Wissenschaftslehre por parte de Marianne Weber, donde este texto es incluido. La recopilación de las ediciones posteriores realizadas por Johannes Winckelmann incluye los siguientes doce escritos: 1. "Roscher und Knies und die logischen Probleme der historischen Nationalökonomie"; 2. "Die «Objektivität» sozialwissenschaftlicher und sozialpolitischer Erkenntnis"; 3. "Kritische Studien auf dem Gebiet der kulturwissenschaftlichen Logik"; 4. "R. Stammlers «Überwindung» der materialistischen Geschichtsauffasung"; 5. "Nachtrag zu dem Aufsatz über R. Stammlers «Überwindung» der materialistischen Geschichtsauffasung"; 6. "Die Grenznutzlehre und das «psychophysische Grundgesetz»"; 7. «Energetische» Kulturtheorien"; 8. "Über einige Kategorien der verstehende Soziologie"; 9. "Die drei reinen Typen der legitimen Herrschaft"; 10. "Der Sinn der «Wertfreiheit» der soziologischen und ökonomischen Wissenschaften"; 11. "Soziologische Grundbegriffe"; 12. "Wissenschaft als Beruf".

5 H. H. Bruun \& S. Whimster, "Introduction”, M. Weber, Collected Methodological Writings, London \& New York, Routledge, 2012, pp. xi-xxviii, aquí p. xvi. Si hacemos de este texto (“«Energetische» Kulturtheorien”) el último de la serie, entonces habría que excluir de los escritos metodológicos la reseña de 1909 del libro de Alfred Weber.

6 Los siete primeros textos los doce que componen los Gesammelte Aufsätze zur Wissenschaftslehre.

7 Señalo las ediciones en castellano más accesibles de estos siete primeros textos: M. Weber, "Roscher y Knies y los problemas lógicos de la escuela histórica de economía", M. Weber, El problema de la irracionalidad en las ciencias sociales (ed. de José María García Blanco), Madrid, Taurus, 2001, pp. 3-173; M. Weber, La “objetividad" del conocimiento en la ciencia social y en la política social (ed. de Joaquín Abellán), Madrid, Alianza, 2009, que también se encuentra en M. Weber, "La «objetividad» cognoscitiva de la ciencia social y de la política social", M. Weber, Ensayos sobre metodología sociológica (trad. de José Luis Etcheverry), Buenos Aires, Amorrortu, 2006, pp. 39-101; M. Weber, "Estudios críticos sobre la lógica de las ciencias de la cultura", M. Weber, Ensayos sobre metodología sociológica (trad. de José Luis Etcheverry), Amorrortu, Buenos Aires, 2006, pp. 102-174; M. Weber, La “superación” de la concepción materialista de la historia. Crítica a Stammler (trad. de Cecilia Abdo), Barcelona, Gedisa, 2014; "M. Weber, "La teoría de la utilidad marginal y la «ley fundamental de la psicofísica», M. Weber, El problema de la irracionalidad en las ciencias sociales (ed. de José María García Blanco), Madrid, Taurus, 2001, pp. 174-192. El único texto de los siete que faltaba por traducir es precisamente el presentamos aquí. 
En esos años, Weber escribe y publica los dos artículos de La ética protestante y el espíritu del capitalismo, las respuestas a los críticos de estos dos artículos, el artículo sobre las sectas, múltiples artículos sobre la cuestión educativa, sobre cuestiones agrarias del presente y del pasado, sobre la bolsa, sobre Rusia, sobre y para la Verein für Sozialpolitik, la Psicofisica del trabajo industrial, etc., por nombrar algunos no todos- de sus textos de ese período. No se trata, por tanto, de una fase exclusivamente metodológica del pensamiento de Weber, sino de un período de especial dedicación a estas cuestiones ${ }^{8}$. Sin embargo, esta dedicación se lleva a cabo de forma peculiar: si excluimos el texto sobre la "objetividad", los escritos metodológicos se desarrollan sobre todo en forma de crítica - e incluso reseña - de otros textos y autores con motivo de acontecimientos ocasionales como la publicación de un libro relevante. El carácter propositivo solamente destaca en el texto sobre la "objetividad", mientras que los demás textos (Roscher y Knies, Stammler, Meyer, Brentano), así como el que aquí presentamos (Ostwald), tienen un carácter netamente polémico. Mediante la crítica y el enfrentamiento Weber establece algunos aspectos centrales de su propuesta "metodológica", aunque siempre de forma fragmentaria, abierta y sin aspiración a la unidad, como si Weber sugiriera posibles desarrollos sistemáticos que él no pudiera o no quisiera recorrer. Por eso, apoyándose en reflexiones de otros, apunta con mucho esfuerzo a varias ideas no del todo conectadas entre sí. Como si se tratara de una sucesión de exabruptos, Weber ofrece en los escritos metodológicos del período 1903-1909 varias respuestas incompletas y parciales ante los problemas que ponían en el centro del debate público otros académicos como los anteriormente citados. Dejando que los términos del debate fueran fijados por los académicos cuyas obras reseña o cuyas figuras enjuicia ${ }^{9}$, domina sus textos un tono áspero que también caracteriza a otros textos de ese período que abordan cuestiones completamente diferentes, como la respuesta a los críticos de La ética protestante y el espiritu del capitalismo o los artículos sobre la cuestión educativa. Por estas razones Teorías "energéticas" de la cultura comparte con el resto de escritos metodológicos del período anteriormente citado - con la consabida excepción de escrito sobre la "objetividad" - la sequedad de la prosa, la exigua elaboración de la escritura y la chocante falta de coherencia interna ${ }^{10}$, pues en muchas ocasiones el autor salta de un tema a otro sin razón evidente generando la impresión de que el texto estuviera escrito a disgusto. Como es obvio, estas características hacen del texto una lectura difícil y dolorosa.

Por otra parte, las reflexiones metodológicas no acaban en la selección de escritos del volumen sobre Wissenschaftslehre. Tomando sólo en consideración textos escritos durante el período mencionado, podríamos incluir como parte de la "metodología" varios pasajes muy conocidos de otros escritos, como por ejemplo pasajes de las últimas páginas de La ética protestante y el espíritu del capitalismo, e incluso varios pasajes no tan conocidos de escritos como las respuestas a los críticos de la mencionada obra.

9 No hay que desligar esta incitación al debate y a la polémica de su compromiso con el Archiv für Sozialwissenschaft und Sozialpolitik, donde con la salvedad del escrito sobre Roscher y Knies aparecen los textos publicados en vida por Weber sobre metodología de los años 1903-1909. El artículo sobre la Objetividad (n. 19, 1904, 2287), Meyer (n. 22, 1906, 143-207), Stammler (n. 24, 1907, 94-151), Brentano (n. 27, 1908, 546-558), Ostwald (n. 29, 1909, 575-598) e incluso la reseña del libro de Adolf Weber (n. 29, 1909 615-620). Se trata de un período de fuerte compromiso con la revista.

10 Características habitualmente destacadas por los traductores de estos textos al castellano, al inglés, al francés o al italiano. 


\section{La "metodología" weberiana}

Sabemos entonces que Teorías "energéticas" de la cultura pertenece a los escritos metodológicos de Weber de un determinado período (1903-1909). Sin embargo, ¿qué es la metodología en general para Weber? Al esbozar una sucinta respuesta a esta pregunta hemos de considerar diversos aspectos que, por lo general, se encuentran ligados a la historia de la recepción de la obra de Weber ${ }^{11}$.

a) Weber no publicó una Wissenschaftslehre ni un volumen que recogiera sus escritos metodológicos. Aunque se han encontrado en su correspondencia con su editor varios indicios de su interés por publicar un volumen sobre lógica o metodología, no parece probable que estuviera interesado en publicar un volumen sobre estas cuestiones. Fue Marianne Weber quien publicó en 1922 el volumen sobre Wissenschaftslehre ${ }^{12}$ - un término con fuertes resonancias filosóficas, sobre todo fichteanas. La razón principal del desinterés de Weber consiste en su particular consideración de los problemas metodológicos.

b) Weber siempre tuvo un interés instrumental por la metodología ${ }^{13}$ : para él se trata de una reflexión a posteriori sobre los procedimientos que fueron útiles para llegar a resultados concretos. Por sí misma la reflexión metodológica no lleva a ninguna parte. No obstante, esto no significa que los escritos metodológicos sean una cuestión accesoria en el pensamiento de Weber, pues al estar vinculados con las disputas de su tiempo que atraviesan su obra muestran parte de lo esencial de su pensamiento.

c) "Metodología" ha significado cosas muy diferentes desde principios de siglo XX en adelante. Los escritos de metodología de Weber se insertan en discusiones en el ámbito de la historia, de la historia económica ${ }^{14} \mathrm{y}$ de las ciencias

11 Como ha sido puesto de relieve por numerosos estudiosos de la obra de Weber. Véase por ejemplo para el ámbito en lengua castellana A. Morcillo Laíz, "La dominación filantrópica. La Rockefeller Foundation y las ciencias sociales en español (1938-1973)", A. Morcillo Laíz \& E. Weisz (eds.), Max Weber en Iberoamérica. Nuevas interpretaciones, estudios empíricos y recepción, México, FCE, 2016, pp. 573-605; sobre la cuestión de la recepción del papel de la historia, véase P. Piedras Monroy, Max Weber y la crisis de las ciencias sociales, Tres Cantos, Akal, 2004, pp. 9-41.

12 La edición de los escritos metodológicos de Marianne Weber y Johannes Winckelmann recoge los siete textos anteriormente citados del período 1903-1909, así como cinco textos posteriores: los dos ensayos publicados en Logos en 1913 (sobre la sociología interpretativa) y en 1917 (sobre la Wertfreiheit), el texto póstumo sobre los tres tipos de dominación legítima, los conceptos sociológicos fundamentales y la famosísima Ciencia como vocación.

13 W. Hennis, "Estar libre de valores como un precepto de distanciamiento", ARBOR 539-540, 1990, pp. 11-27, aquí p. 19, recoge varias formulaciones en este sentido por parte de Weber que paso a citar: "ojalá desaparezca muy pronto la moda actual de que los trabajos de los principiantes tengan que ir adornados con investigaciones gnoseológicas"; "la metodología (sólo) puede ser una autorreflexión sobre los medios que han probado su eficacia en la práctica, y que tener una conciencia explícita de éstos no (es) un requisito para un trabajo fructífero como no lo es el conocimiento de la anatomía para caminar «correctamente»"; "las ciencias sólo se fundamentaron con la presentación y la solución de problemas objetivos y su método se sigue desarrollando; las consideraciones puramente metodológicas o gnoseológicas, por el contrario, nunca han tomado parte en esto de una manera decisiva".

14 Esta es la perspectiva defendida por F.. Tenbruck, "Max Weber und Eduard Meyer", en W. Mommsen \& J. Osterhammel (Hrsg.), Max Weber und seine Zeitgenossen, Göttingen \& Zürich, Veröffentlichungen des Deutschen Historischen Instituts London, 1988, pp. 337-379, aquí p. 342 y ss. También hay una versión inglesa de este mismo texto: F. Tenbruck, "Max Weber and Eduard Meyer", W. Mommsen \& J. Osterhammel (eds.), Max Weber and his Contemporaries, London, Allen \& Unwin, 1987, pp. 234-267, aquí p. 238. Una excelente descripción 
culturales en general ${ }^{15}$, remitiendo a contextos alejados de nuestros intereses actuales y de los de los sociólogos norteamericanos que convirtieron a Weber en el padre de la sociología. Si se leen estos textos desde una metodología de las ciencias sociales tal como se configuran en la segunda mitad del siglo XX, entonces resultan ininteligibles y no sirven para nada. Y así han permanecido durante mucho tiempo, especialmente fuera de Alemania ${ }^{16}$. La imagen de un Weber fundador de la sociología coloca estos textos en un segundo plano ${ }^{17}$. Estos textos no son ensayos de metodología sociológica, sino que proceden de las querellas disciplinares alemanas que desgarraban las ciencias de la cultura desde finales del siglo XIX. Una fecunda comprensión de los escritos metodológicos de Weber obliga a situarlos en las disputas de su tiempo y a poner en claro los contextos habitualmente no explicados de donde surge en última instancia la propuesta de la Wertfreiheit ${ }^{18}$.

d) Ahora bien, la recepción de los escritos de metodología fuera de Alemania ha solido proceder de esta interpretación/incomprensión propia de la sociología. No es por tanto una casualidad que hayan sido sobre todo académicos alemanes — con mayor o menor acierto — ${ }^{19}$ quienes más hayan trabajado

de ese contexto se encuentra en J. L. Villacañas, "Weber y el ethos del presente", M. Weber, La ética protestante y el espíritu del capitalismo, Tres Cantos, Akal, 2013, pp. 7-73, aquí pp. 10-32.

15 Aunque - a mi juicio - sean más relevantes los estudios que enfatizan la importancia de las discusiones en el ámbito de la historia y de la historia económica - y que son los menos-, la influencia del neokantismo ha sido bastante trabajada por los sociólogos y los autores de habla inglesa. Véase por ejemplo los estudios metodológicos de T. Burger, Max Weber's Theory of Concept Formation, Duke, Duke University Press, 1987; G. Oakes, Weber and Rickert. Concept Formation in the Cultural Sciences, Cambridge \& London, MIT Press, 1988; F. Ringer, Max Weber's Methodology. The Unification of the Cultural and Social Sciences, Cambridge \& London, Harvard University Press, 1997; o S. Eliaeson, Max Weber's Methodologies, Cambridge, Polity, 2002; S. Whimster, Understanding Weber, London \& New York, Routlegde, 2007; H. H. Bruun, Science, Values and Politics in Max Weber's Methodology, London, Ashgate, 2007, entre muchos otros. Sobre los estudios culturales, véase especialmente L. Scaff, Fleeing the Iron Cage. Culture Politics and Modernity in the Thought of Max Weber, Berkeley - Los Angeles - London, University of California Press, $1989 \mathrm{o}$ Ch. Turner, Modernity and Politics in the Work of Max Weber, London \& New York, Routledge, 1992.

16 Por esta razón es por la que, por ejemplo la influyente monografia de R. Bendix Weber. An Intellectual Portrait (traducida al castellano como R. Bendix. Max Weber, Buenos Aires, Amorrortu, 1979) no menciona en absoluto estos escritos, así como tampoco lo hace con las conferencias sobre la política y la ciencia como vocación. Véase E. Gavilán, "De las ventajas e inconvenientes del resentimiento para el estudio de las religiones: Nietzsche y Weber", J. Llinares (coord.) Nietzsche, 100 años después, Valencia, Pretextos, 2002, pp. 135-154, aquí p. 138 y ss.

17 Como bien muestra la terca incomprensión de Parsons del principio de la Wertfreiheit y la desatención con respecto a estos debates por parte de los editores de estos escritos en lengua inglesa. Entre los muchos estudios existentes, véanse especialmente las correcciones que realizan W. Hennis, Max Webers Fragestellung. Studien zur Biographie des Werks, Tübingen, J. C. B. Mohr (Paul Siebeck), 1987; W. Mommsen, "Max Weber in Modern Social Thought", W. Mommsen, The Political and Social Theory of Max Weber. Collected Essays, Cambridge \& Oxford, Polity, 1989, pp. 169-196; P. Lassman \& I. Velody (eds.), Max Weber's Science as a Vocation, London, Unwin Hyman, 1989; y F. Gil Villegas, "Importancia de las cuatro respuestas de Max Weber a sus primeros críticos", M. Weber, La ética protestante y el espiritu del capitalismo, México, FCE, 2011, pp. 502-538.

18 W. Hennis, "Estar libre de valores como un precepto de distanciamiento", op. cit., p. 13; J. Abellán, "Estudio preliminar", M. Weber, Por qué no se deben hacer juicios de valor en la sociología y en la economía, Alianza, Madrid, 2010, pp. 11-62, aquí pp. 11-19; P. Josephson, "Lehrfreiheit, Lernfreiheit, Wertfreiheit: Max Weber and the University Teachers' Congress in Jena 1908", Max Weber Studies 4, 2, pp. 201-219.

19 En muchas ocasiones, sobre todo von Schelting y Henrich, tienden a destacar erróneamente — a mi juicio — la unidad de la metodología weberiana. Véase H. H. Bruun, Science, Values and Politics in Max Weber's Methodology, op. cit., p. 3. 
los textos sobre metodología, desde von Schelting (1934), Henrich (1952), Tenbruck (1959) y Weiss (1975) hasta Hennis (1987) y Wagner y Zipprian $(1994)^{20}$. Para la joven sociología norteamericana y la empresa intelectual de Parsons y sus seguidores, estos textos eran inútiles, pues remitían a un contexto oscuro y desconocido: los debates sobre la educación sostenidos en foros muy diversos, los debates en el seno del Verein für Sozialpolitik y las disputas sobre el estatuto de las ciencias culturales, las discusiones sobre la investigación histórica, sobre la lógica de los conceptos, sobre el papel del científico, etc. Esta vía, que conducía a la imagen de un Weber demasiado imbuido en las preocupaciones de su época, dificultaba la creación de un sabio universal fundador de la moderna sociología científica. A tal efecto, para comprender los escritos metodológicos hay que recomponer la imagen de Weber, poniendo de relieve su faceta de estudioso del Derecho, historiador de la economía, experto en política social, etc. No procede ni tampoco resulta oportuno desentrañar en esta breve presentación los complejos vericuetos de los contextos y los debates en que surgen los escritos metodológicos ${ }^{21}$, pero queremos dejar apuntado, sin embargo, que sin ellos no se comprende el sentido último de su "metodología".

e) Las ediciones con la traducción de los escritos metodológicos siguen siendo demasiado sociológicas. La versión inglesa de los Gesammelte Aufsätze zur Wissenschaftslehre (The Methodology of the Social Sciences, traducido en 1949 por Edward A. Shils y Henry A. Finch) sólo recoge tres escritos: el de la "objetividad", la crítica de Meyer y el escrito sobre la Wertfreiheit. Aparte de otras traducciones de textos sueltos por parte de otros autores, fue Guy Oakes quien se encargó de traducir en los años setenta varios textos de la compilación de Marianne Weber que no estaban incluidos en la edición inglesa de 1949 como el de Roscher y Knies (1975) o el de Stammler (1977). La edición italiana de Pietro Rossi de 1958 sólo recoge cuatro escritos: el escrito sobre la "objetividad", la crítica de Meyer, el ensayo sobre las categorías de 1913 y el escrito sobre la Wertfreiheit. La edición francesa de Julien Freund de 1965 y la edición española traducida por José Luis Etcheverry recogen los mismos textos que la edición italiana ${ }^{22}$. En estos idiomas se han ido produciendo progresivamente traducciones de los textos de los Gesammelte Aufsätze zur Wissenschaftslehre no recogidos en dichas ediciones, pero lo que mancomuna a estas

20 A. v. Schelting, Max Webers Wissenschaftslehre, Tübingen, J. C. B. Mohr (Paul Siebeck), 1934; D. Henrich, Die Einheit der Wissenschaftslehre Max Webers, Tübingen, J. C. B. Mohr (Paul Siebeck), 1952; F. Tenbruck, "Die Genesis der Methodologie Max Webers”, Kölner Zeitschrifts für Soziologie und Sozialpsychologie 11, 1959, pp. 573-630; J. Weiß, Max Webers Grundlegung der Soziologie, München, Sauer, 1975; W. Hennis, Max Webers Fragestellung. Studien zur Biographie des Werks, Tübingen, J. C. B. Mohr (Paul Siebeck), 1987; G. Wagner \& H. Zipprian (Hrsg.), Max Webers Wissenschaftslehre. Interpretation und Kritik, Frankurt am Main, Suhrkamp, 1994.

21 Véase la ya citada edición de Gerhard Wagner y Heinz Zipprian que recoge una serie de estudios sobre la Wissenschaftslehre weberiana, especialmente su introducción: G. Wagner \& H. Zipprian, "Zur Einführung”, G. Wagner \& H. Zipprian (Hrsg.), Max Webers Wissenschaftslehre. Interpretation und Kritik, Suhrkamp, Frankurt am Main, 1994, pp. 9-28. Por otra parte, una selección de escritos de la época sobre la cuestión de la Wertfreiheit se encuentra en H. H. Nau (Hrsg.), Der Werturteilsstreit, Metropolis, Marburg, 1996.

22 M. Weber, Saggi sul metodo delle scienze storico-sociali (ed. Pietro Rossi), Torino, Eunaudi, 2001; M. Weber, Essais sur la téorie de la science (ed. Julien Freund), Paris, Plon, 1965; M. Weber, Ensayos sobre metodología sociológica (trad. de José Luis Etcheverry), Buenos Aires, Amorrortu, 2006. 
ediciones canónicas de los grandes textos sobre la metodología weberiana es, por lo general, la mirada sociológica. Hasta la mejor y más completa edición de estos escritos en un idioma diferente al alemán (la excelente edición inglesa de Bruun y Whimster de 2012) adolece de esta mirada principalmente sociológica.

f) Solamente algunos estudios, llevados a cabo principalmente por académicos alemanes, han conseguido iluminar ese fondo de donde proceden los textos metodológicos weberianos ${ }^{23}$. En última instancia, podríamos afirmar que lo que se ha denominado cuestiones de metodología no son sino cuestiones normativas, especialmente ligadas a los debates disciplinarios de la época, que en muchos sentidos siguen siendo los nuestros.

\section{Novedad, interés e ideas centrales del texto}

La característica más novedosa del texto que aquí presentamos ${ }^{24}$ es que se trata del único escrito "metodológico" en el que Weber debate con un científico ajeno a las ciencias de la cultura. Mientras que en el resto de escritos del período mencionado (1903-1909) todos los querellados (Roscher, Knies, Meyer, Stammler y Brentano) pertenecen a este último ámbito, la reseña del libro de Ostwald constituye el único "diálogo" con un científico natural. Ahora bien, Weber no debate con Ostwald sobre cuestiones químicas —ámbito en el que Ostwald recibió un Premio Nobel一, sino sobre cuestiones de las ciencias de la cultura. ${ }^{25} \mathrm{Y}$ no entra en polémica con Ostwald con la intención de defender, frente a la tendencia expansionista de los científicos naturales, su ámbito científico en tanto que propio. No se trata de una cuestión de "poder" o de separación de espacios, sino de comprensión de la lógica y la metodología de las ciencias de la cultura y, en definitiva, de la ciencia en general - especialmente cuando se ocupa de asuntos prácticos vinculados al deber ser.

Este breve escrito nos muestra un Weber disciplinar y disciplinario, que no tiene reparos en amonestar a un "compañero" que intenta aplicar acríticamente los postulados "energéticos" a un ámbito donde éstos no funcionan, dando lugar a tesis tan

23 F. Tenbruck, además del artículo de 1959 ya citado, ha desarrollado esta cuestión en varios artículos como "Das Werk Max Webers: Methodologie und Sozialwissenschaften" o "Die Wissenschaftslehre Max Webers" recogidos en el volumen F. Tenbruck, Das Werk Max Webers. Gesammelte Aufsätze zu Max Weber (Hrsg. Harald Homann), Tübingen, J. C. B. Mohr (Paul Siebeck), 1999, así como en el artículo F. Tenbruck, “Abschied von der «Wissenschaftslehre»", J. Weiß (Hrsg.), Max Weber heute. Erträge und Probleme der Forschung, Frankfurt am Main, Suhrkamp, 1999.

24 Los destinos editoriales de este escrito son curiosos. Considerado como un texto menor dentro del volumen de los Gesammelte Aufsätze zur Wissenschaftslehre, no ha sido traducido en ninguna de las ediciones de los textos metodológicos en otros idiomas anteriormente citadas, salvo en la reciente edición inglesa, que sí lo incluye: M. Weber, Collected Methodological Writings (ed. H. H. Bruun \& S. Whimster) London \& New York, Routledge, 2012. Jon Mark Mikkelsen y Charles Schwartz también realizaron una traducción de este escrito que fue publicada en 1984 en la Mid-American Review of Sociology (M. Weber, "«Energetic» Theories of Culture", Mid-American Review of Sociology 12, 1984, 9.2., pp. 33-58). Aparte de éstas, no conozco otras traducciones. Más arriba hemos aventurado varias razones que explican esta ausencia: es un escrito menor (una reseña), de difícil lectura, con pocos desarrollos teóricos y que aborda unas cuestiones (el energetismo) y un autor (Ostwald) completamente olvidados desde hace mucho tiempo.

25 Dice Weber: "en la medida en que se menciona el ámbito de problemas económicos y político-sociales, me referiré a algunas páginas sobre este aspecto". Infra, p. 454. 
estrafalarias que Weber no desaprovecha la ocasión para ridiculizar. No se enfrenta aquí a historiadores, representantes de la Nationalökonomie y científicos de la cultura, predecesores o contemporáneos, en su particular lucha metodológica, sino a un científico natural orgulloso y exitoso en su propio ámbito que, sin embargo, defiende un modelo erróneo y periclitado sobre la especificidad (o más bien la ausencia de la misma) de las ciencias de la cultura. Ante la ingenua pero dolorosa arrogancia del científico natural que entra en el terreno de las ciencias de la cultura como un elefante en una cacharrería, Weber opta por una durísima crítica que, en términos generales, se despliega en dos direcciones: en el respecto lógico y en el empírico o fáctico. En relación con el respecto lógico, la crítica de Weber a Ostwald se fundamenta en un aspecto puramente metodológico: Ostwald funge como representante de una extendida posición en el seno de las ciencias naturales que ignora la especificidad de las ciencias culturales. En relación con el respecto empírico o fáctico, la crítica de Weber a Ostwald se fundamenta en la tesis sobre lo que acabará siendo la Wertfreiheit: Ostwald funge como representante de una extendida posición en el seno de las ciencias naturales — pero también culturales- que niega la pertinencia de la distinción entre juicios de valor y datos científicos.

a) En relación con el aspecto lógico hemos de distinguir a su vez dos momentos: por un lado, la crítica de la interpretación "energética" de la cultura propia de Ostwald; por otro lado, la crítica del programa de unificación de la metodología científica - un programa más amplio, sobre el que Ostwald se apoya. Es evidente que el motivo que desencadena esta furibunda y mordaz reseña no es otro que la extralimitación de Ostwald, quien aplica sus postulados "energéticos" a la vida cultural. Cruel y mordaz, Weber denuncia esta irresponsable actuación. Sin embargo, Weber valora positivamente el movimiento de acercamiento entre las ciencias naturales y las ciencias culturales y llega a sostener que la labor de formación de conceptos puede ser enriquecida mediante la experimentación en otros campos. Ahora bien, semejante acercamiento debe estar precedido por la comprensión de las especificidades de cada grupo de ciencias. Y así como los científicos de la cultura en general saben que no pueden imponer sus conceptos y su metodología a los científicos naturales, Weber reclama que ocurra lo mismo en sentido inverso. No obstante, dado lo extendido de la opinión que defiende que las ciencias culturales habrían de funcionar en la formación de conceptos abstractos del mismo modo como funcionan las ciencias naturales, Weber lanza su diatriba contra esa condescendencia — procedente en el fondo de la ignorancia- de los científicos naturales:

Pero en vista de la arrogancia desmedida con la que algunos representantes de las ciencias naturales parecen ocuparse del trabajo de otras disciplinas (históricas, principalmente), de disciplinas que siguen otros caminos que corresponden a otros objetivos metódicos, es necesario hacer constar que también es válido para un pensador tan importante como Ostwald el duodécimo mandamiento de Chwolson ${ }^{26}$.

Weber no se muestra indulgente con Ostwald, pues este mandamiento del físico ruso Chwolson prohíbe escribir sobre temas de lo que no se sabe lo suficiente. Weber no rechaza un intercambio con los científicos naturales, pero defiende que el presu-

$26 \quad$ Infra, p. 466. 
puesto básico de una sana colaboración pasa por el reconocimiento de la especificidad de cada conjunto de disciplinas.

El principal escollo para dicha colaboración consiste en la absolutización del proceso de formación de conceptos de las ciencias naturales como modelo científico único; una absolutización que conduce al desprecio de otras ciencias cuya formación de conceptos se realiza de forma diferente. La interpretación "energética" de los asuntos culturales se sustenta sobre la hipóstasis del modelo cientifista, que según Weber da lugar a engendros como el libro de Ostwald que reseña. Mediante su agria crítica Weber pretende que los científicos naturales dejen de arrogarse la capacidad - a su juicio, errónea e injustificada - de poder decir algo con sentido sobre asuntos culturales sólo porque ya han alcanzado una metodología científica segura en su ámbito. El propio Weber señala que reseña este libro, no porque sea especialmente bueno o especialmente malo - aunque sea más bien lo segundo-, sino porque es ejemplar en su error y porque constituye un tipo.

Sin embargo, Ostwald constituye un eslabón más en la larga cadena de propuestas que buscaban alcanzar la unidad de la metodología científica. Weber tiene claro que dicha unidad es imposible y contraproducente en su praxis, llegando a afirmar, por ejemplo, que es indiferente para la teoría económica si en la astronomía domina el paradigma ptolemaico o el copernicano. La validez de la teoría económica no depende del éxito de las ciencias naturales, pues no existe una jerarquía de las ciencias: la validez de las ciencias de la cultura — que según muchas propuestas metodológicas unitarias y jerárquicas estarían en la cúspide - no está subordinada a la validez de las ciencias naturales - que a su vez estarían en la base-. Weber pone de relieve que el inspirador de esta insensata consideración para Ostwald es Comte, hacia el cual sólo tiene reproches:

Por eso solamente hace falta detenerse un instante en estas utopías para darse cuenta de lo que, a pesar de todo, siempre olvidan todas las modernas teorías del método: que la jerarquía de las ciencias de Comte es un esquema ajeno a la vida (lebensfremd Schema) de un grandísimo pedante que no comprende que existen disciplinas con objetivos de conocimiento totalmente diferentes, cada una de las cuales, derivadas de ciertas experiencias cotidianas inmediatas, debe elaborar y sublimar el contenido de este conocimiento "no científico" bajo puntos de vista completamente distintos y absolutamente independientes ${ }^{27}$.

Estas disquisiciones, enmarcadas en grandes disputas metodológicas, quizás nos parezcan — con razón - lejanas y aburridas porque las consideramos superadas ${ }^{28}$. No obstante, la cuestión más relevante para Weber se halla, no en la intromisión de los científicos naturales en los temas y métodos propios de las ciencias culturales, sino en su crítica de la introducción de postulados valorativos en el propio quehacer científico - con lo que llegamos al respecto empírico o fáctico de la crítica de Ostwald.

28 Podríamos resumir esta parte de la crítica de Weber haciéndole decir algo así como "callen los científicos-naturales en asuntos ajenos, respeten la especificidad de las ciencias de la cultura". 
b) En relación con el respecto empírico o fáctico, "esta confusión de juicios de valor (Werturteilen) y ciencia empírica aflora por todas partes de la manera más catastrófica" 2 . Lo más dañino para la ciencia no es para Weber la situación de "inferioridad" que los científicos de la cultura puedan llegar a sentir con respecto a los científicos naturales, sino la alegre introducción de juicios de valor en el quehacer científico por parte de científicos naturales, pero también por parte de científicos de la cultura. No se trata ahora de la necesaria toma de conciencia de la especificidad de las ciencias de la cultura, sino de la exclusión — en general - de los juicios de valor del quehacer científico. Y en esta cuestión de la constante intromisión de los postulados valorativos se encuentran hermanados por igual los científicos naturales y los científicos de la cultura.

Esta reseña presenta $-\mathrm{y}$ en estas cuestiones resulta muy interesante - varias formulaciones del hiato entre el ser y el deber ser y, además, ejemplifica una de las múltiples y fatales confusiones entre ambos planos mediante el análisis - en clave paródica - de algunas de las opiniones, a veces extravagantes, de Ostwald. Weber se recrea en la denuncia del ropaje supuestamente científico con el que este brillante químico cubre sus opiniones; unas opiniones tan lícitas como las de cualquier otro ser humano, pero absolutamente ilícitas cuando Ostwald pretende fundamentarlas "científicamente", pues mediante ese artificio hace pasar como conocimiento "científico" su propia opinión.

La máxima weberiana que impide la deducción de juicios de valor a partir de datos científicos queda ilustrada mediante un hábil desmontaje de la constante intromisión, por parte de Ostwald, de sus postulados preferidos en ámbitos tan diferentes como el arte, el lenguaje, el derecho y la educación. Según Weber, las consideraciones energéticas resultan inverosímiles aplicadas al arte y se detiene jocosamente ante diversas posibilidades, todas ellas ridículas: un cuadro eficiente energéticamente podría ser uno que represente batallas y explosiones, quizá un cuadro realizado con la máxima rapidez o una serie de cuadros prefabricados o de recetas de cocina, etc. El punto de vista energético, de acuerdo con Weber, no llega a tocar lo más básico del valor del arte: "iqué infortunio que el «arte» comience donde el «punto de vista» del técnico acaba!"30. Parecidas monstruosidades teóricas resultan de la aplicación del punto de vista energético al lenguaje. Desde las reflexiones de Ostwald resulta imposible comprender la riqueza del lenguaje natural y de figuras como la ambigüedad, mientras que su única y enloquecida propuesta consistiría en la creación de un lenguaje formal que anulara las impurezas e imprecisiones del lenguaje natural. Donde Weber se muestra más ácido es en las consideraciones sobre la teoría energética aplicada al Derecho. Según Weber, Ostwald introduce sin saberlo ideales de distinto tipo bajo su supuesta consideración puramente energética: defiende una igualdad ante la ley bastante peculiar - pues prevé castigos diferentes en función de la posición social-, asume ideas del pensamiento retributivo, desarrolla consideraciones propias del iusnaturalismo, etc. Aparte de la intromisión de estas consideraciones claramente no energéticas, resulta que de un seguimiento estricto de su teoría energética se deducirían situaciones extremadamente cómicas, como la condena a sufrir severos castigos para aquellos que no mejoran la proporción eficiente (como jubila-

Infra, p. 464.

30 Infra, p. 462. Hay que apuntar que algunos párrafos de este texto anticipan las consideraciones sobre el progreso del arte que aparecen en el escrito de 1917 sobre la Wertfreiheit. 
dos, filólogos e historiadores) y penas de tipo corporal o físico para aquellos que sí la mejoran, pero transgreden la ley. Por último, Weber no da crédito ante la propuesta de Ostwald de defender la libertad de pensamiento y de opinión como una conquista científica. Estas libertades no son el producto de ideales tecnológicos o científicos - nos dice Weber-, del mismo modo que las motivaciones para dedicarse a la ciencia, e incluso el sentido mismo de la labor científica, no quedan constreñidos a ideales energéticos o puramente científicos: "una ciencia natural en absoluto debería pretender estar autorizada a dar una respuesta determinante a esta pregunta práctica, pero es aún menos evidente cómo podría deducirse de una «proporción energética eficiente» algún deber ético en relación a uno u otro comportamiento" ${ }^{31}$.

En resumidas cuentas: según Weber, por un lado, no se pueden aplicar los conceptos y métodos de las ciencias naturales en las ciencias de cultura - en caso contrario se producen engendros como el libro de Ostwald-, y, por otro, hemos de guardarnos ante la intromisión de postulados prácticos en la labor científica. En esta reseña furibunda, errática en su escritura y un tanto caprichosa en ocasiones - como si estuviera escrita a vuelapluma, pero precisamente por ello caracterizada por un estilo ágil y descuidado, quizá demasiado-- Weber se muestra preocupado por la falta de respeto ante la ciencia en general, y ante las ciencias de la cultura en particular - , unas ciencias que deberían constituirse como el espacio dedicado al análisis de los juicios de valor, no a la introducción de los mismos como supuesta verdad científica por parte de miopes científicos naturales que solamente proyectan sus postulados prácticos en un reino donde más bien deberían permanecer en silencio.

$31 \quad$ Infra, p. 464. 